\title{
Distribución corporal e inserción de ácaros (Pterygosomatidae: Neopterygosoma) en la lagartija Liolaemus pacha (Iguania: Liolaemidae)
}

\author{
Body Distribution and Insertion of Mites (Pterygosomatidae: \\ Neopterygosoma) in Liolaemus pacha Lizard (Iguania: Liolaemidae)
}

\footnotetext{
Viviana Isabel Juárez Heredia**; María Daniela Miotti ${ }^{2}$;

Marcela Beatriz Hernández ${ }^{3}$; Cecilia Inés Robles ${ }^{1}$; Monique Halloy ${ }^{\dagger}$

1 Instituto de Comportamiento Animal, Fundación Miguel Lillo, Miguel Lillo 251, (4000) San Miguel de Tucumán, Argentina.vijuarez@lillo.org.ar

2 Programa de Investigación de la Biodiversidad Argentina (PIDBA), Facultad de Ciencias Naturales e Instituto Miguel Lillo, Miguel Lillo 205, Tucumán, Argentina.

3 Instituto de Fisiología Animal, Fundación Miguel Lillo, Miguel Lillo 251, (4000) San Miguel de Tucumán, Argentina.
}

\section{RESUMEN}

Los ácaros suelen ser ectoparásitos comunes de los vertebrados. Su distribución corporal puede variar entre especies y pueden ocasionar lesiones en la piel. Los ácaros de Liolaemus pacha pertenecen al género Neopterygosoma y se ubican por debajo de las escamas de su hospedador. Los escasos registros sobre la relación ectoparásitohospedador nos llevó a proponernos como objetivos, definir cuantitativamente la distribución corporal y determinar los posibles daños ocasionados por los ácaros en los sitios de inserción mediante estudios histopatológicos. Para el conteo de ácaros se definieron cinco regiones corporales y se realizaron cortes histológicos de las zonas de la piel que presentaban ácaros. En total, se analizaron 52 ejemplares de ambos sexos. Se observó una mayor concentración de ácaros en la región ventral, específicamente en los laterales del vientre y en la región gular. El análisis histológico mostró que no hay daños epidérmicos ni reacciones inflamatorias en la piel del hospedador. Por lo

Ref. bibliográfica: Juárez Heredia, V. I.; Miotti, M. D.; Hernández, M. B.; Robles, C. I.; Halloy, M. "Distribución corporal e inserción de ácaros (Pterygosomatidae: Neopterygosoma) en la lagartija Liolaemus pacha (Iguania: Liolaemidae)". Acta zoológica lilloana 64 (1): 1-12. Fundación Miguel Lillo, Tucumán, Argentina. D.O.I.: https://doi.org/10.30550/j.azl/2020.64.1/1

> Recibido: 20 de diciembre de 2019 - Aceptado: 6 de abril de 2020

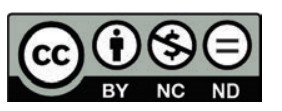

> URL de la revista: http://actazoologica.lillo.org.ar

- Esta obra está bajo una Licencia Creative Commons Atribución - No Comercial - Sin Obra Derivada 4.0 Internacional. 
tanto, concluimos que los ácaros de $L$. pacha no producen daños locales en la piel, e incluso, su forma característica de inserción, pone en duda el tipo de alimentación hematófaga, reportada en otros estudios. Los resultados obtenidos aportan nueva información sobre la biología de Liolaemus pacha y Neoptorygosoma sp., permitiendo mejorar la interpretación de su interacción.

Palabras clave - Histología, parásitos, interacción ectoparásito-huésped, infestación.

\section{ABSTRACT}

Mites are usually common ectoparasites of vertebrates. Their body distribution can vary between species and can cause skin lesions. Liolaemus pacha mites belong to the genus Neopterygosoma and are located below the scales of its host. The few records on the ectoparasite-host relationship led us to propose ourselves as objectives, quantitatively define the body distribution and determine the possible damage caused by the mites at the insertion sites through histopathological studies. For the mite count, five body regions were defined and histological sections of the skin areas presenting mites were made. A total of 52 individuals of both sexes were analyzed. A higher concentration of mites was observed on the flanks and in the gular region. Histological analysis showed that there is not epidermal damage or inflammatory reactions in the host's skin. Therefore, we conclude that $L$. pacha mites do not cause local skin damage and even their characteristic form of insertion cast doubt on the type of hematophagous feeding reported in other studies. The results obtained provide new information about the biology of Liolaemus pacha and Neoptorygosoma $s p$., allowing to improve the interpretation of their interaction.

Keywords - Histology, parasites, ectoparasites-host interaction, infestation.

\section{INTRODUCCIÓN}

Los parásitos son organismos que viven en o sobre un hospedador, obteniendo de él la totalidad o parte de sus nutrientes, causando algún tipo de daño o enfermedad a su hospedador (Price, 1980). Los ácaros suelen ser ectoparásitos comunes de los vertebrados, incluyendo a numerosos reptiles y entre ellos las lagartijas. Los ácaros suelen distribuirse en zonas y/o estructuras corporales específicas, entre las que se destacan los pliegues o "bolsas" axilares (hundimiento y prolongación de la piel), inguinales y post-femorales (Loveridge, 1951; Bertrand y Modrý, 2004; García de la Peña et al., 2004; Gomes de Carvalho et al., 2006; Delfino et al., 2011) y regiones como cuello, vientre, patas y cola (Bochkov et al., 2009; Espinoza Carniglia et al., 2015, 2016).

Los ectoparásitos pueden producir efectos perjudiciales sobre el hospedador como lesiones en la piel, inflamación o anemia (Klukowski, 2004; Bulté et al., 2009). Goldberg y Bursey (1991), describen los efectos a nivel histológico de cuatro especies diferentes de ectoparásitos: Neotrombicula califórnica (Trombiculidae), Geckobiella 
texana (Pterygosomatidae), Ophionyssus natricis (Macronyssidae) e Ixodes pacificus (Ixodidae) sobre el tegumento de la lagartija Uta stansburiana (Phrynosomatidae). Solo $N$. califórnica e I. pacificus provocaron daños tegumentarios y respuestas inflamatorias en los tejidos de su hospedador, mientras que no se registraron lesiones histológicas sobre hospedadores parasitados por $O$. natricis y $G$. texana, aunque en este último se encontró en el macerado, rastros de eritrocitos de su hospedador.

Actualmente, son numerosos los estudios sobre la presencia de ácaros en lagartijas de diferentes países (e.g. Schall et al., 2000; Klukowski, 2004; Martin et al., 2008; Carvalho et al., 2006; Rocha et al., 2008; Rubio y Simonetti, 2009; Espinoza Carniglia et al., 2016; Jackson y Bateman, 2018), sin embargo, existen sólo tres trabajos que describen la presencia de ácaros en especies de lagartijas del género Liolaemus (Dittmar de la Cruz et al., 2004; Fajfer y Gonzalez Acuña, 2013; Juárez Heredia et al., 2014).

Los ácaros encontrados en Liolaemus pacha pertenecen al género Neopterygosoma (Pterygosomatidae), los cuales son considerados ectoparásitos permanentes, partenogenéticos, especie-específicos y viven principalmente debajo de las escamas del hospedador (Juárez Heredia et al., 2014; Fajfer, 2019). Liolaemus pacha no presenta estructuras especializadas como los "bolsillos" o pliegues de la piel para la inserción de los ácaros, pero sí se determinó que hay regiones con mayor intensidad de infestación (Juárez Heredia et al., 2014), las mismas fueron definidas de forma descriptiva a través del análisis de fotos. Hasta el momento no se han realizado estudios que describan los puntos de inserción de Neopterygosoma y los posibles daños o respuestas inflamatorias sobre su hospedador.

Debido a que la relación entre Liolaemus pacha y sus ácaros es importante desde el punto de vista ecológico y evolutivo para ambas especies, nos proponemos los siguientes objetivos: investigar si cuantitativamente existe un patrón específico de la distribución de los ácaros sobre L. pacha; y analizar histopatológicamente el área de inserción de los ácaros en la piel de su hospedador, a fin de corroborar si éstos ocasionan algún tipo de daño en el tegumento o reacción inflamatoria local.

\section{MATERIALES Y MÉTODOS}

La población de Liolaemus pacha proviene de la localidad de Los Cardones ( $26^{\circ} 40^{\prime}$ $1,5^{\prime}$ S, $65^{\circ} 49^{\prime}$ 5, ' " W, $2700 \mathrm{msnm}$ ), ubicada a $20 \mathrm{~km}$ al este de la comuna de Amaicha del Valle, departamento de Tafí del Valle, Tucumán. Realizamos visitas mensuales durante los periodos de actividad de las lagartijas, desde octubre de 2013 hasta febrero de 2015. Se capturaron lagartijas adultas con la técnica de lazo corredizo, la cual consiste en usar una caña de aproximadamente $1,5 \mathrm{~m}$ en cuyo extremo se ata un lazo corredizo de hilo. Los individuos capturados se mantuvieron en bolsas de tela para su posterior análisis. De cada ejemplar se registró el sexo y la presencia de ácaros. Para contabilizar el número de ácaros en las lagartijas infestadas, se definieron cinco regiones corporales ventrales: región gular (RG), patas delanteras (PD), patas traseras (PT), región lateral (RL) y cola (C) (Fig. 1). Cabe destacar, que durante los muestreos preliminares solamente se encontraron ácaros en la región ventral. El conteo 


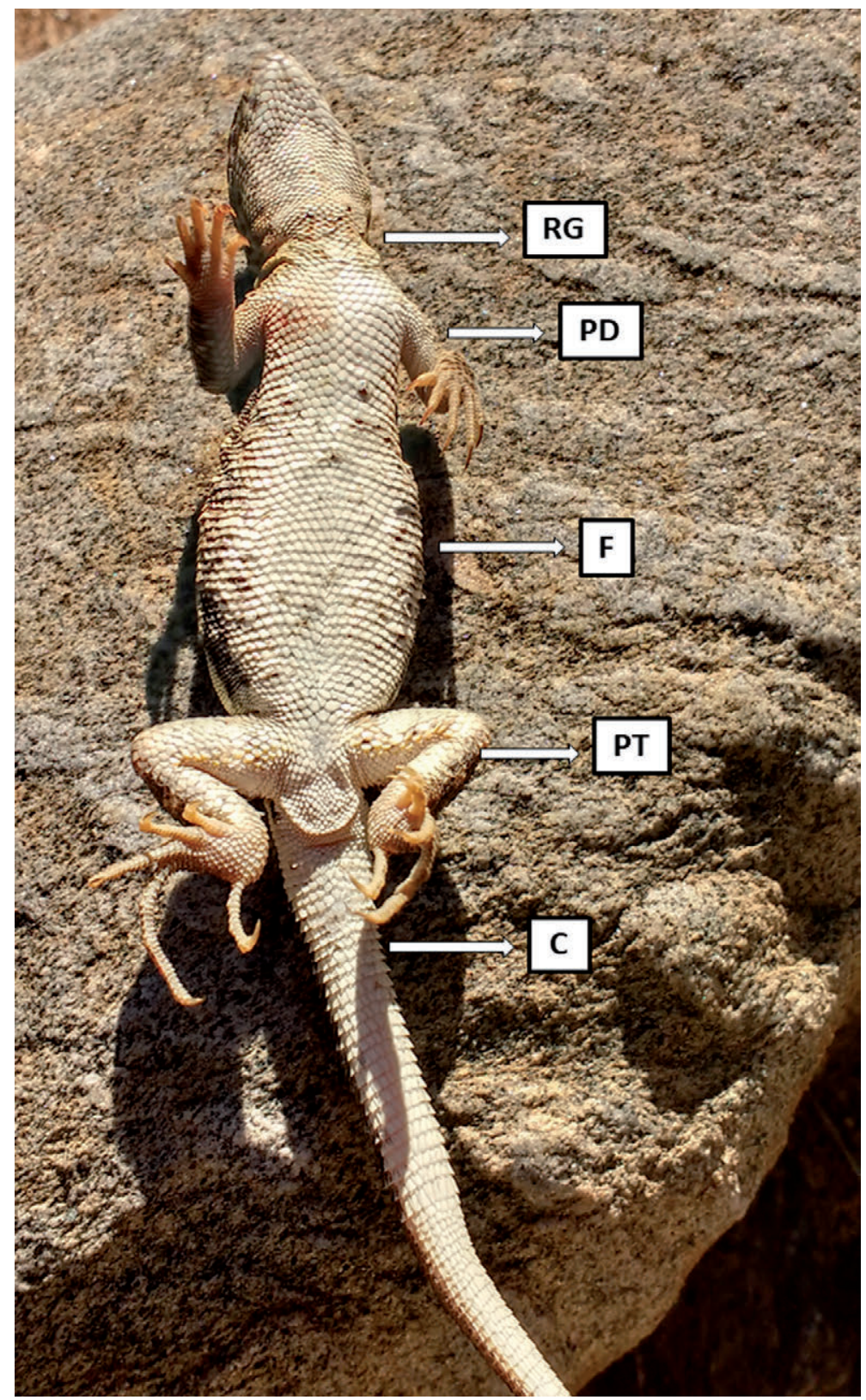

Figura 1. Vista ventral de un macho de L. pacha donde se indican las cinco regiones corporales definidas. RG: región gular, PD: patas delanteras, F: flancos, PT: patas traseras, C: cola.

Figure 1. Ventral view of a male L. pacha where the five defined body regions are indicated. RG: gular region, PD: front legs, F: flanks, PT: hind legs, C: tail.

se realizó utilizando un microscopio (marca Wild Heerbrugg, precisión 10x/21) y se tomaron fotografías con cámara digital (Samsung PL 120) para registrar las zonas con mayor concentración de ácaros. Para la recolección de ácaros, con un hisopo humedecido con alcohol 70 \% previamente se rozó suavemente las zonas donde el ácaro estaba inserto lo que facilitó la extracción sin dañar las muestras. Los ácaros fueron removidos con una aguja de tuberculina y colocados en eppendorf con alcohol al $70 \%$. Los ectoparásitos muestreados fueron montados con alcohol polivinilico (PVC) y solución de Hoyer (Krantz, 1970) para su posterior identificación. 
Para el análisis estadístico de la intensidad de ectoparásitos en las cinco regiones corporales definidas, se utilizó la Prueba de Friedman $(\mathrm{p}=0,05)(\mathrm{InfoStat}$ Di Rienzo et al., 2015). Posterior a la toma de todos los datos, cada lagartija fue liberada en su sitio exacto de captura.

Para llevar a cabo los estudios histológicos del tegumento, se trabajó con dos machos adultos los cuales tenían cada uno más de 50 ácaros en la región ventral del cuerpo. Ambas lagartijas murieron de forma natural no especifica durante el muestreo por lo que se aprovechó el material para su estudio (material ingresado a la colección de Herpetología, Fundación Miguel Lillo). De cada uno de los ejemplares se tomaron muestras de piel de las zonas con más cantidad de ácaros. Los parches de piel $(10 \times 10 \mathrm{~mm})$ fueron procesados según las técnicas de rutina para microscopia óptica (McManus y Mowry, 1968; Ham, 1975). Los cortes de $6 \mu \mathrm{m}$ de espesor fueron coloreados con hematoxilinaeosina y estudiados con microscopio óptico (marca Zeiss Axiolab y Primo Star). Las descripciones histológicas de la piel y sus componentes se realizaron siguiendo a Genneser (1999) y Ross y Pawlina (2007), mientras que las microfotografías fueron tomadas con cámara digital (marca AxioCam ERC5S) y digitalizadas con el software ZEN-Blue (Edition 2012).

\section{RESULTADOS}

Se capturó un total de 52 lagartijas infestadas por Neopterygosoma (35 machos y 17 hembras). Teniendo en cuenta las cinco regiones corporales definidas para determinar la presencia de ácaros, se encontraron diferencias significativas con respecto a la intensidad de infestación, siendo la región $\mathrm{F}$ (flancos) la que presentó mayor intensidad o carga de ácaros, seguida de la RG (Regón Gular), C (Cola), PD (Patas Delanteras) y PT (Patas Traseras) $(\mathrm{n}=$ 52; $\mathrm{T}^{2}=19,7 ; \mathrm{p}=0,0001$ ) (Fig. 2).

$\mathrm{El}$ análisis histológico mostró que los ácaros fueron encontrados alojados en una cámara, formada entre dos escamas superpuestas sobre la piel del ejemplar, tratándose así de un ectopa-

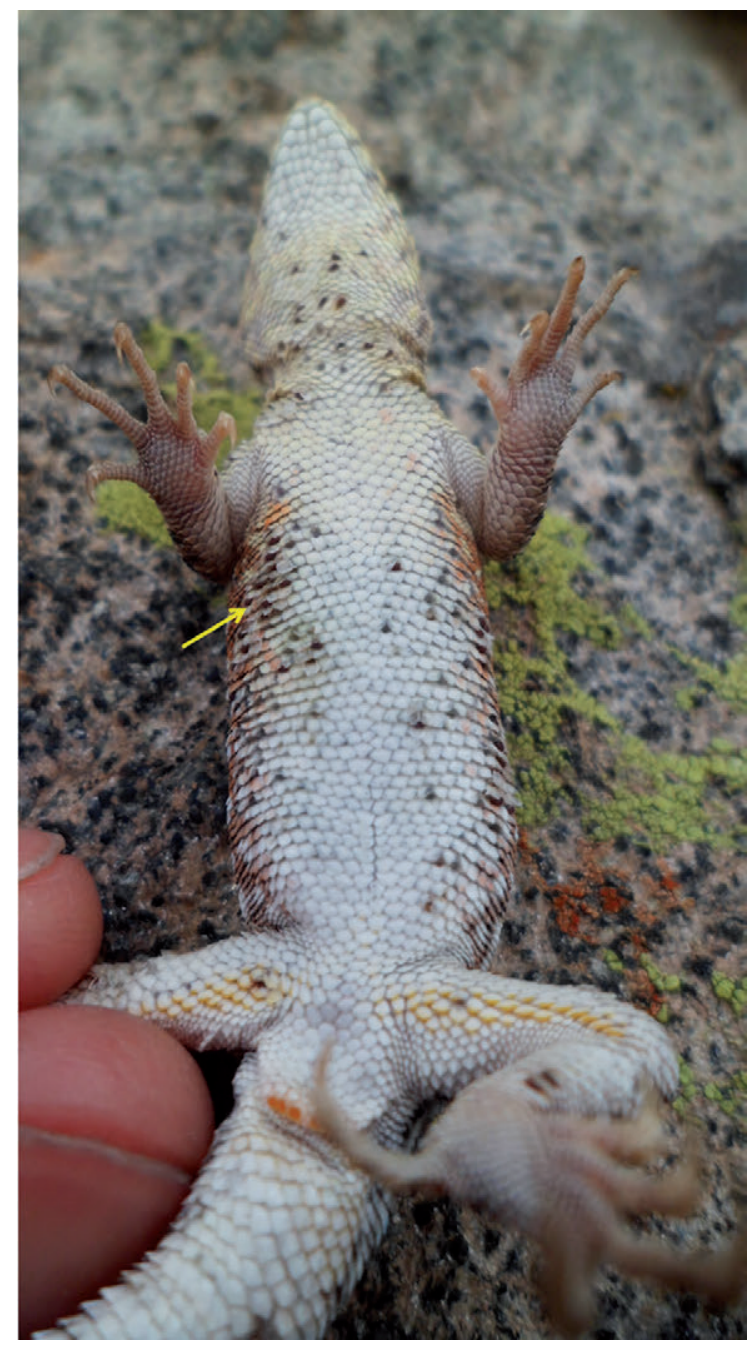

Figura 2. Región ventral de un macho de L. pacha con mayor intensidad de infestación en la región de los flancos (punta de flecha amarilla señala un ácaro).

Figure 2. Ventral region of a male $L$. pacha with the highest intensity of infestation in the region flanks (yellow arrowhead points to a mite). 


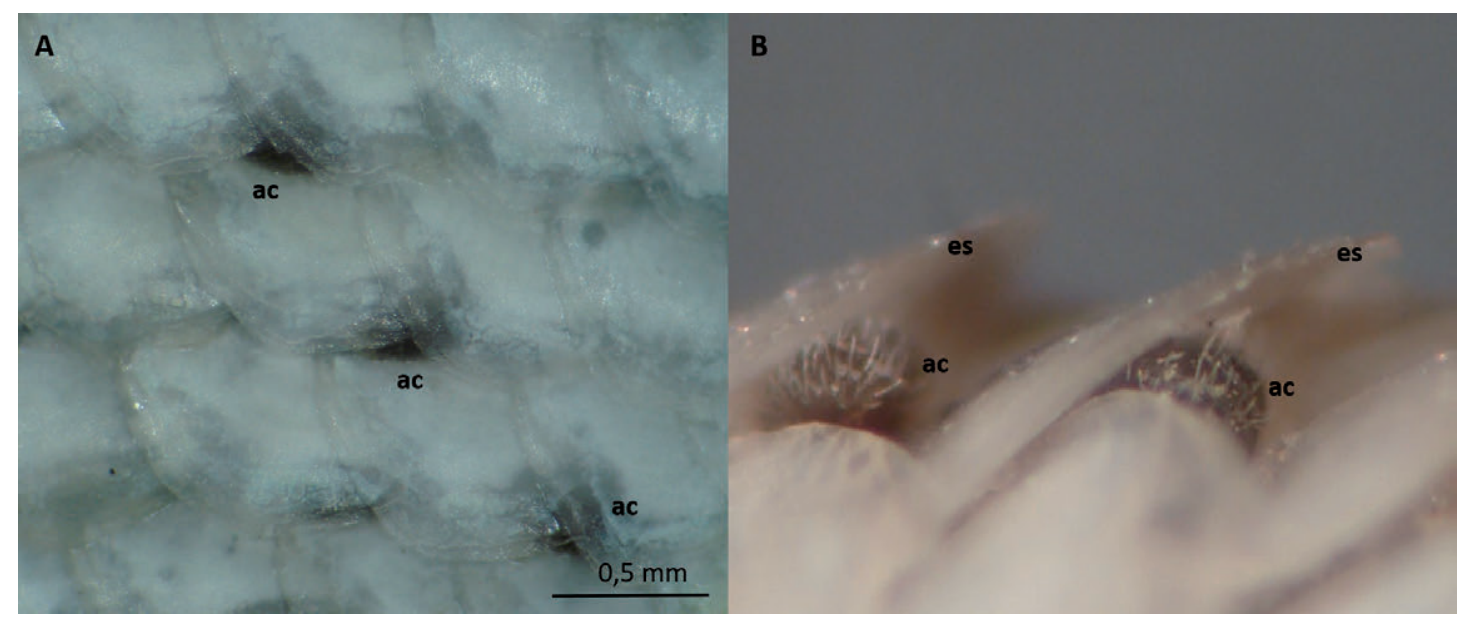

Figura 3. Ectoparásitos supraepidérmicos en: A) Vista frontal de las escamas de la región del vientre. B) Vista lateral de las escamas del vientre. ac: ácaro; es: escama.

Figure 3. Supraepidermal ectoparasites in: A) Frontal view of the scales of the belly region. B) Lateral view of the scales of the belly. ac: mite; is: flake.

rásito supraepidérmico (Figs. 3 y 4B). En las zonas de la piel que no contenían ácaros no presentaron este espacio o cámara entre las escamas (Fig. 4A).

No se encontraron ácaros insertos o sujetos con sus piezas bucales al tegumento, ni daños o perforaciones en la epidermis. Tanto la epidermis como la dermis, presentaban aspecto normal y no se observaron reacciones inflamatorias, queratomas $o$ presencia de células del sistema inmune. Por lo que a nivel histopatológico, los ácaros no ocasionaron ningún tipo de daño en la piel del hospedador en el lugar específico de inserción ni en sus alrededores (Fig. 4A).

\section{DISCUSIÓN}

La mayoría de los géneros de la familia Pterygosomatidae, se caracterizan por ubicarse debajo de las escamas de su hospedador, en especial en la región ventral (Bertrand, 2002; Dittmar de la Cruz et al., 2004; Fajfer, 2012; Bertrand et al., 2013; de Oliveira et al., 2019). A diferencia de la lagartija Liolaemus pictus, en la cual se encontraron ácaros en el dorso del cuerpo (Pterygosomatidae: Neopterygosoma Espinoza Carniglia et al., 2016), en L. pacha sólo se registraron ácaros en la zona ventral. La distribución ventral y la ubicación especifica de los ácaros debajo de las escamas formando cámaras en la piel del hospedador, reflejan estrategias por parte del ectoparásito para disminuir los riesgos de su eliminación por comportamientos de rascado o fricción de alguna parte del cuerpo de la lagartija. Por otro lado, la distribución ventral de los ácaros evitaría la incidencia directa del sol y aumenta el contacto cercano con el suelo, lo que permitiría mantener las condiciones de humedad y protección para su supervivencia (Clopton y Gold, 1993).

Las escamas imbricadas son una de las características morfológicas presentes en los lagartos que favorecen la inserción de ectoparásitos (Menezes et al., 2011). La 


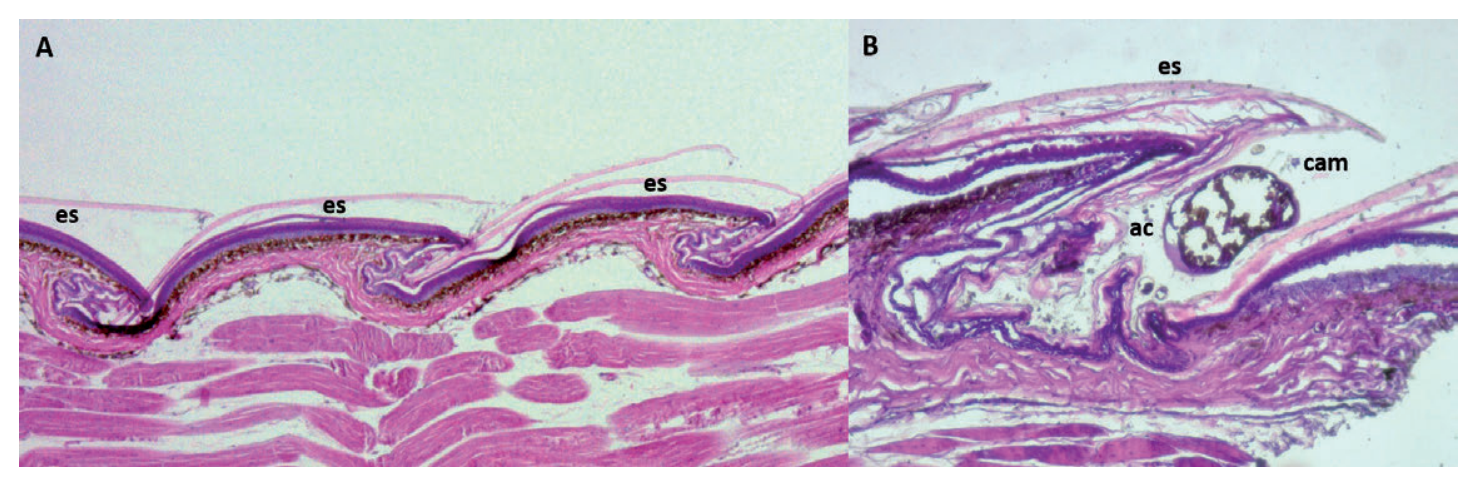

Figura 4.: Piel del vientre. A) sin ácaros y sin cámaras entre las escamas. B) con ácaros y cámara. ac: ácaro; cam: cámara; es: escama.

Figure 4. Belly skin. A) without mites and without cameras between the scales. B) with mites and camera. ac: mite; cam: camera; es: flake.

región ventral de L. pacha se caracteriza por tener este tipo de escamas, e incluso son más flexibles que las del dorso, lo cual favorecería la inserción de los Neopterygosoma Este resultado también fue registrado en Tropidurus torquatus infestado por Eutrombicula alfreddugesi (Cunha Barros y Rocha, 2000; Cunha Barros et al., 2003).

Goldberg y Bursey (1991), proponen que el tipo de distribución de un ectoparásito estará definido de acuerdo con el tiempo que pase sobre su hospedador, es decir, los ácaros que infestan cortos periodos de tiempo se ubicarían en zonas corporales de fácil acceso; mientras que aquellos que infestan largos periodos de tiempo o son permanentes, se encontrarían en zonas corporales más protegidas, como son los “bolsillos" o pliegues. Según Fajfer (2019), Neopterygosoma son reportados como ectoparásitos permanentes, por lo que el desarrollo de "bolsillos" o pliegues de la piel favorecen esta característica (Bertrand y Modrý, 2004). Sin embargo, en L. pacha no se registraron estas estructuras y los ácaros se encontraron debajo de las escamas del hospedador. El género Liolaemus, pertenece al clado Pleurodonta, el cual desde el punto de vista evolutivo, es considerado un grupo relativamente nuevo (surgió hace 10 millones de años), a diferencia del clado Acrodonta (30 millones de años) (Townsend et al., 2011; Blankers et al., 2013). Townsend et al. (2011), postulan que Pleurodonta muestra una rápida y temprana explosión de diversificación de especies y variaciones morfológicas temporales, lo que también afectó a la evolución de sus ácaros. Esto explicaría la ausencia de estructuras especializadas en Liolaemus pacha para contener sus ácaros. Sin embargo, Simoes et al. (2015) postulan que Acrodontas y no acrodontas estuvieron en contacto en el Cretácico Tardío en América del Sur (resultado en base a un registro fósil de un ejemplar de Gueragama sulamericana de Brasil) pero fueron desplazados por exclusión competitiva por los no acrodontas. Consideramos importante los estudios sistemáticos y filogenéticos en el género Liolaemus por su amplia distribución y numerosas especies, para poder determinar factores que determinan la coexistencia entre ectoparásito hospedador y posibles efectos evolutivos para la adaptación en la infestación.

Teniendo en cuenta los trabajos que definen como ectoparásitos permanentes a Neopterygosoma, consideramos que el ciclo de vida del ácaro debe ser estudiado de 
forma completa para confirmar su total permanencia sobre un hospedador. La mayor intensidad de ácaros definida en la región de los flancos y posteriormente en la región gular, confirman lo obtenido en Juárez Heredia et al. (2014). La distribución corporal diferenciada en regiones en Liolaemus pacha también fue registrada en otras especies de lagartijas (Bertrand et al., 1995; Cunha Barros y Rocha, 1995; Cunha Barros et al., 2003; Bochkov et al., 2009; García de la Peña et al., 2010; Delfino et al., 2011; Espinoza Carniglia et al., 2016).

En estudios realizados en las especies de lagartijas Uta stansburiana y Sceloporus jarrovii (Goldberg y Bursey, 1991; Goldberg y Hulshuh, 1992) se observaron reacciones inflamatorias alrededor de las zonas de inserción de las piezas bucales de ectoparásitos de la familia Trombiculidae e Ixodidae. También se reportaron en los puntos de inserción, precipitados acelulares, proteínas coaguladas extendidas en la dermis y presencia de granulomas (Goldberg y Bursey, 1991; Goldberg y Hulshuh, 1992). En este estudio, Liolaemus pacha no presentó histológicamente ningún tipo de daño ni reacciones inflamatorias en el tegumento, debajo de las escamas que contenían ácaros. Estos resultados son coincidentes con los reportados en Uta stansburiana, infestado por Geckobiella texana (Pterygosomatidae, Goldberg y Bursey, 1991).

La mayoría de los géneros de la familia Pterygosomatidae son hematófagos o se alimentan de linfa (Bertrand et al., 2013). Sin embargo, el género Neopterygosoma, fue recientemente descripto para lagartijas de la familia Liolaemidae y su tipo de alimentación aún no fue definida (Fajfer, 2019). Teniendo en cuenta estas referencias y los resultados de este trabajo, la ausencia de reacciones inflamatorias y las adaptaciones epidérmicas para albergar a los ácaros, concluimos que los mismos no serían hematófagos, pudiéndose alimentar de líquido intersticial (com. pers. Dr. Ricardo Paredes León)

Consideramos que los resultados obtenidos sobre la relación ectoparásito-hospedador son importantes, teniendo en cuenta la escasa y desactualizada información sobre el tema (e.g. Goldberg y Bursey, 1991; Goldberg y Hulshuh, 1992). La ausencia de efectos histopatológicos en esta relación ectoparásito-hospedador, aporta información sobre el tipo de interacción que existe y las adaptaciones para mantener este tipo de parasitismo. Estos estudios aportan información valiosa sobre la biología de las especies y son de suma importancia para comprender las relaciones ecológicas y co-evolutivas entre parásito-hospedador.

\section{AGRADECIMIENTOS}

Agradecemos a la Dra. Monique Halloy por ser fuente de inspiración diaria, por habernos marcado un camino y guiarnos por él. Gracias al Dr. Ricardo Paredes León por la identificación de los ácaros. Agradecemos a Pablo Corrales por su colaboración en los muestreos de campo. Gracias a la Dirección de Flora, Fauna silvestre y suelo de Tucumán, por otorgarnos el permiso de colecta (Res. $\mathrm{N}^{\circ}$ 169-13). 


\section{FINANCIAMIENTO}

Los viajes de campos fueron financiados bajo el marco del proyecto del Instituto de Comportamiento Animal ( ${ }^{\circ}$ Z004912), Fundación Miguel Lillo, y por la beca de Finalización de Doctorado 2015 de CONICET (Resolución No 4971).

\section{PARTICIPACIÓN}

La primera autora realizó los viajes de campo al sitio de estudio, la toma de muestras (identificación y conteo de ácaros) y la escritura del manuscrito. La Dra. Daniela Miotti realizó los preparados histológicos e interpretación de los mismos. La Dra. Marcela B. Hernández y Dra. Cecilia I. Robles han contribuido en la revisión y corrección del manuscrito.

\section{CONFLICTOS DE INTERÉS}

En el presente trabajo no existe conflicto de interés entre las autoras.

\section{LITERATURA CITADA}

Bertrand, M. (2002). Morphologic adaptations to parasitism on reptiles: Pterygosomatidae (Prostigmata: Raphignathina). En Acarid phylogeny and evolution: adaptation in mites and ticks. Springer Netherlands, 233-240.

Bertrand, M., Modrý, D. (2004). The role of mite pocket-like structures on Agama caudospinosa (Agamidae) infested by Pterygosoma livingstonei sp. n. (Acari: Prostigmata: Pterygosomatidae). Folia Parasitologica, 51: 61-66.

Bertrand, M., Paperna, I., Finkelman, S. (1995). Etude préliminaire du genre Pterygosoma Peters, 1849 (Actinedida: Pterygosomidae): compléments à la description de Pterygosoma bibronii Jack, 1962 et notes sur les caractères évolutifs des Pterygosomidae. Acarologia, 36: 133-143.

Bertrand, M., Kukushkin, O., Pogrebnyak, S. (2013). A new species of mites of the genus Geckobia (Prostigmata, Pterygosomatidae), parasitic on Mediodactylus kotschyi (Reptilia, Gekkota) from Crimea. Vestnik Zoologii, 47(2): 1-13.

Bochkov, A. V., Melnikov, D. A., Nazarov, R. A. (2009). Pterygosoma pseudotrapelus sp. nov. (Acariformes: Pterygosomatidae) ectoparasite of Pseudotrapelus sinaitus (Squamata: Agamidae) from Jordan. Zootaxa, 2232: 61-68.

Bulté, G., Plummer, A. C.; Thibaudeau, A., Blouin Demers, G. (2009). Infection of yarrow's spiny lizards (Sceloporus jarrovii) by chiggers and malaria in the chiricahua mountains, Arizona. The Southwestern Naturalist, 54: 204-207.

Blankers, T., Townsend, T. M., Pepe, K., Reeder, T. W., Wiens, J. J. (2013). Contrasting global-scale evolutionary radiations: phylogeny, diversification, and mor- 
phological evolution in the major clades of iguanian lizards. Biological Journal of the Linnean Society, 108: 127-143.

Carvalho, R. L., Antoniazzi, M. M., Jared, C., Silva, A. M. J., Santos, A. A., Egami, M. I. (2006). Morphological, cytochemical, and ultrastructural observations on the blood cells of the reptile Tupinambis merianae (Squamata). Comparative Clinical Pathology, 15(3): 169-174.

Clopton, R. E., Gold, R. E. (1993). Distribution and seasonal and diurnal activity patterns of Eutrombicula alfreddugesi (Acari: Trombiculidae) in a forest edge ecosystem. Journal of Medical Entomology, 30(1): 47-53.

Cunha Barros, M., Rocha, C. F. D. (1995). Parasitismo por ácaros Eutrombicula alfreddugesi (Trombiculidae) em duas espécies simpátricas de Mabuya (Sauria: Scincidae): o efeito do habitat na prevalência e intensidade parasitária. Oecologia Brasiliensis, 1: 307-316.

Cunha Barros, M., Rocha, C. F. D. (2000). Ectoparasitism by chigger mites (Eutrombicula alfreddugesi: Trombiculidae) in a restinga lizard community. Ciência e Cultura, 52(2): 108-113.

Cunha Barros, M., Van Sluys M., Vrcibradic, D., Galdino, C. A. B., Hatano, F. H., Rocha, C. F. D. (2003). Patterns of infestation by chigger mites in four diurnal lizard species from a resting habitat (Jurubatiba) of Southeastern Brazil. Brazilian Journal of Biology, São Carlos, 63(3): 393-399.

Delfino, M. M. S., Ribeiro, S. C., Furtado, I. P., Anjos, L. A., Almeida, W. O. (2011). Pterygosomatidae and Trombiculidae mites infesting Tropidurus hispidus (Spix, 1825) (Tropiduridae) lizards in northeastern Brazil. Brazilian Journal of Biology, 71(2): 549-555.

de Oliveira, C. N., Campos, I. H. M. P., de Oliveira, J. B., de Moura, G. J. B. (2019). Acari of lizards from Atlantic Forest in northeastern Brazil. Neotropical Biology and Conservation, 14: 109.

Di Rienzo, J. A., Casanoves, F., Balzarini, M. G., Gonzalez, L., Tablada, M., Robledo, C. W. (2015). InfoStat versión 20151. Córdoba: Universidad Nacional de Córdoba. Available from: www.infostat.com.ar

Dittmar de la Cruz, K., Morando, M., Avila, L. (2004). Description of a New pterygosomatid mite (Acari, Actinedida: Pterygosomatidae) parasitic on Liolaemus spp. (Iguania: Liolaemini) from Argentina. Zootaxa, 521: 1-6.

Espinoza Carniglia, M., de La Fuente, M. S., Pérez, A., Victoriano, P. F., Salas, L. M. (2015). Fragmented host distribution and trombiculid parasitic load: Eutrombicula araucanensis and Liolaemus pictus in Chile. Acarologia, 55(2): 209-217.

Espinoza Carniglia, M., Pérez Leiva, A., Silva de la Fuente, M. C., Victoriano Sepúlveda, P., Moreno Salas, L. (2016). Abundancia y distribución de ácaros parásitos (Eutrombicula araucanensis y Pterygosoma sp.) en lagartijas (Liolaemus pictus) de Chile central. Revista Mexicana de Biodiversidad, 87(1): 101-108.

Fajfer, M. (2012). Acari (chelicerata) parasites of reptiles. Acarina, 20(2): 108-129.

Fajfer, M. (2019). Systematics of reptile-associated scale mites of the genus Pterygosoma (Acariformes: Pterygosomatidae) derived from external morphology. Zootaxa, 4603(3): 401-440. 
Fajfer, M., Gonzalez Acuña, D. G. (2013). Pterygosomatid mites of a new species group ligare (Acariformes: Pterygosomatidae: Pterygosoma) parasitizing tree iguanas (Squamata: Liolaemidae: Liolaemus). Zootaxa, 3693(3): 301-319.

García de la Peña, C., Contreras Balderas, A., Castañeda, G., Lazcano, D. (2004). Infestación y distribución corporal de la nigua Eutrombiculida alfredduguesi (Acari: Trombiculidae) en el lacertilio de las rocas Sceloporus couchi (Sauria: Phrynosomatidae). Acta Zoológica Mexicana (n.s), 20(2): 159-165.

García de la Peña, C., Gadsden, H., Salas Westphal, A. (2010). Carga ectoparasitaria en la lagartija espinosa de Yarrow (Sceloporus jarrovii) en el cañón de las Piedras Encimadas, Durango, México. Interciencia, 35(10): 772-776.

Genneser, F. (1999). Histología. Editorial Médica Panamericana. 813.

Goldberg, S. R., Bursey, C. R. (1991). Integumental lesions caused by ectoparasites in a wild population of the side-blotched lizard (Uta stansburiana). Journal of Wildlife Diseases, 27: 68-73.

Goldberg, S. R., Holshuh, H. J. (1992). Ectoparasite induced lesions in mite pockets of the Yarrow's spiny lizard, Sceloporus jarrovii (Phrynosomatidae). Journal of wildlife diseases, 28(4): 537-541.

Gomes de Carvalho, A. L., de Araújo, A. F., Silva, H. R. D. (2006). Patterns of parasitism by Eutrombicula alfreddugesi (Oudemans) (Acari, Trombiculidae) in three species of Tropidurus Wied (Squamata, Tropiduridae) from cerrado habitat of central Brazil. Revista Brasileira de Zoologia, 23(4): 1010-1015.

Ham, A. W. (1975). Tratado de histología. $7^{a}$ ed. Editorial Interamericana.

Jackson, L. N., Bateman, H. L. (2018). Differing ectoparasite loads, sexual modes, and abundances of whiptail lizards from native and non-native habitats. Herpetological Conservation and Biology, 13(1): 294-301.

Juárez Heredia, V. I., Vicente, N., Robles, C., Halloy, M. (2014). Mites in the neotropical lizard Liolaemus pacha (Iguania: Liolaemidae): Relation to body size, sex and season. South American Journal of Herpetology, 9(1): 14-19.

Klukowski, M. (2004). Seasonal changes in abundance of host-seeking chiggers (Acari: Trombiculidae) and infestations on fence lizards, Sceloporus undulatus. Journal of Herpetology, 38: 141-144.

Loveridge, A. (1951). On reptiles and amphibians from Tanganyika territory collected by C.J.P. Ionides. Bulletin ofthe Museum of Comparative Zoology at Harvard College, 106: 177-204.

Martín, J., Amo, L., López, P. (2008). Parasites and health affect multiple sexual signals in male common wall lizards, Podarcis muralis. Naturwissenschaften, 95 (4): 293-300.

McManus, J. F. A., Mowry, R. W. (1968). Técnica Histológica. Atika.

Menezes, V. A., Fontes, A. F., Gettinger, D., Van Sluys, M., Rocha, C. F. D. (2011). A morphometric study of Eutrombicula alfreddugesi (Acari: Trombiculidae) infesting four sympatric species of Tropidurus (Squamata: Tropiduridae) in northeastern Brazil. Phyllomedusa: Journal of Herpetology, 10(1): 79-84.

Price, P. W. (1980). Evolutionary biology of parasites. Princeton, NJ: Princeton University Press. 237. 
Rocha, C. F. D., Cunha Barros, M., Menezes, V. A., Fontes, A. F., Vrcibradic, D., Van Sluys, M. (2008). Patterns of infestation by the trombiculid mite Eutrombicula alfreddugesi in four sympatric lizard species (genus Tropidurus) in northeastern Brazil. Parasite, 15(2): 131-136.

Ross, M. H., Pawlina, W. (2007). Histología. Edición Médica Panamericana.Texto y Atlas Color con Biología Celular y Molecular. 5aed.

Rubio, A. V., Simonetti, J. A. (2009). Ectoparasitism by Eutrombicula alfreddugesi larvae (Acari: Trombiculidae) on Liolaemus tenuis lizard in a Chilean fragmented temperate forest. Journal of Parasitology, 95(1): 244-245.

Schall, J. J., Prendeville, H. R., Hanley, K. (2000). Prevalence of the tick, Ixodes pacificus, on western fence lizards, Sceloporus occidentalis: trends by site, gender, size, season, and mite infestation. Journal of Herpetology, 34: 160-163.

Simões, T. R., Wilner, E., Caldwell, M. W., Weinschütz, L. C., Kellner, A. W. (2015). A stem acrodontan lizard in the Cretaceous of Brazil revises early lizard evolution in Gondwana. Nature Communications, 6(1): 1-8.

Townsend, T. M., Mulcahy, D. G., Noonan, B. P., Sites Jr., J. W., Kuczynski, C. A., Wiens, J.J., Reeder, T. W. (2011). Phylogeny of iguanian lizards inferred from 29 nuclear loci, and a comparison of concatenated and species-tree approaches for an ancient, rapid radiation. Molecular Phylogenetics and Evolution, 61: 363-380. 